\title{
Differential response of Streptococcus mutans towards friend and foe in mixed-species cultures
}

\author{
Correspondence \\ Fengxia Qi \\ Felicia-qi@ouhsc.edu
}

Received 17 January 2011

Revised 3 May 2011

Accepted 6 May 2011

\author{
Jinman Liu,† Chenggang Wu,t‡ I-Hsiu Huang,‡ Justin Merritt \\ and Fengxia Qi
}

\begin{abstract}
College of Dentistry, The University of Oklahoma Health Sciences Center, Oklahoma City, OK 73104, USA
\end{abstract}

\begin{abstract}
In the oral biofilm, the 'mitis' streptococci are among the first group of organisms to colonize the tooth surface. Their proliferation is thought to be an important factor required for antagonizing the growth of cariogenic species such as Streptococcus mutans. In this study, we used a threespecies mixed culture to demonstrate that another ubiquitous early colonizing species, Veillonella parvula, can greatly affect the outcome of the competition between a pair of antagonists such as S. mutans and Streptococcus gordonii. Transcriptome analysis further revealed that $S$. mutans responds differentially to its friend (V. parvula) and foe ( $S$. gordonii). In the mixed culture with $S$. gordonii, all but one of the $S$. mutans sugar uptake and metabolic genes were downregulated, while genes for alternative energy source utilization and $\mathrm{H}_{2} \mathrm{O}_{2}$ tolerance were upregulated, resulting in a slower but persistent growth. In contrast, when cultured with $V$. parvula, S. mutans grew equally well or better than in monoculture and exhibited relatively few changes within its transcriptome. When $V$. parvula was introduced into the mixed culture of $S$. mutans and $S$. gordonii, it rescued the growth inhibition of $S$. mutans. In this three-species environment, $S$. mutans increased the expression of genes required for the uptake and metabolism of minor sugars, while genes required for oxidative stress tolerance were downregulated. We conclude that the major factors that affect the competition between $S$. mutans and S. gordonii are carbohydrate utilization and $\mathrm{H}_{2} \mathrm{O}_{2}$ resistance. The presence of $V$. parvula in the tri-species culture mitigates these two major factors and allows $S$. mutans to proliferate, despite the presence of $S$. gordonii.
\end{abstract}

\section{INTRODUCTION}

Biofilms in the human oral cavity are exceptionally diverse and can harbour more than 800 microbial species (Aas et al., 2005; Keijser et al., 2008; Paster et al., 2001, 2006; Zaura et al., 2009). Dental biofilm formation is hypothesized to be a sequential process (Diaz et al., 2006; Kolenbrander et al., 2006, 2010). After a new tooth emerges or a surface is cleaned, the surface is colonized by a group of bacteria named the 'pioneer colonizers', which are composed mostly of the 'mitis' streptococci (i.e. Streptococcus gordonii, Streptococcus sanguinis, Streptococcus mitis, etc.). Subsequently, early colonizers such as

†These authors contributed equally to this work.

łPresent address: University of Texas HSC-Houston, 6431 Fannin St, MSE R213, Houston, TX 77030, USA.

Abbreviations: CSP, competence-stimulating peptide; MLF, malolactic fermentation; TCA cycle, tricarboxylic acid cycle.

The microarray data discussed in this paper are available from Gene Expression Omnibus (GEO) under accession number GSE28259.

A supplementary table, listing differentially regulated genes in mixed cultures, is available with the online version of this paper.
Streptococcus mutans and veillonellae, and bridging species such as the fusobacteria, join the community through interactions with the pioneer colonizers or by adhering to the available sites on the tooth surface. Growth of the early colonizers then modifies the local environment, making it favourable for the growth of late colonizers, which consist mostly of Gram-negative, obligate anaerobic bacteria. Eventually, through cell growth and co-adhesion, a mature biofilm is formed (for reviews, see Diaz et al., 2006; Kolenbrander et al., 2010). Once formed, the biofilm composition is relatively stable, featuring high biodiversity; however, environmental perturbation can disrupt this ecological balance, leading to the overgrowth of pathogens and the development of oral diseases (Marsh, 1994, 1999, 2006).

The 'mutans' streptococci (mainly S. mutans and Streptococcus sobrinus) are considered primary pathogens for the development of dental caries (tooth decay) (Loesche, 1986), while the mitis streptococci are benign, or even beneficial in preventing dental caries (Becker et al., 2002; Caufield et al., 2000). Both groups of bacteria reside in the same supragingival plaque, have nearly identical nutritional 
requirements and are fierce competitors. Indeed, epidemiological studies have found an inverse relationship between the two groups of bacteria: high numbers of the mutans streptococci correlate with low numbers of the mitis streptococci, while colonization by the latter correlates with delayed colonization by the former (Becker et al., 2002; Caufield et al., 1993, 2000). Likewise, in vivo studies using germ-free rats have revealed a competitive exclusion between S. mutans and S. sanguinis (Mikx et al., 1976). In our in vitro studies, we have shown that bacteriocins (mutacins) produced by $S$. mutans and $\mathrm{H}_{2} \mathrm{O}_{2}$ produced by S. gordonii or S. sanguinis are used as chemical weapons for interspecies competition. The winner of this competition depends upon which species occupies the niche first (Kreth et al., 2005b).

Veillonellae are also early colonizers of the oral biofilm (Hughes et al., 1988, 1992; Palmer et al., 2006), and are one of the most prevalent colonizers of the human oral cavity (Dewhirst et al., 2010). A common characteristic of the genus Veillonella is that they do not utilize carbohydrates; rather, they metabolize lactate, pyruvate and peptones to produce propionate and acetate (Rogosa \& Bishop, 1964). This nutritional requirement makes them dependent upon the streptococci, which excrete lactate as a waste product of carbohydrate fermentation. In vitro and in vivo studies routinely demonstrate veillonellae coaggregating with streptococci (Hughes et al., 1988, 1992; Palmer et al., 2006). Of particular interest in this veillonellae-streptococci association is the removal of lactic acid by the veillonellae. Lactic acid produced by streptococci is primarily responsible for the characteristic demineralization found in dental caries, which raises an interesting question as to the role of veillonellae in cariogenesis. Early studies using gnotobiotic rats found reduced caries activity and tooth demineralization by $S$. mutans when co-inoculated with Veillonella species (Mikx et al., 1972; Mikx \& Van der Hoeven, 1975); however, more recent epidemiological studies in humans have found high numbers of veillonellae associated with high numbers of $S$. mutans in carious lesions (Aas et al., 2008; Becker et al., 2002; Rozkiewicz et al., 2006; Russell et al., 1990). In vitro studies also have found that coculturing S. mutans with Veillonella parvula could increase the resistance of the former to antimicrobial substances (Luppens et al., 2008). In this study, we further explore the molecular mechanism underlying these intricate interspecies relationships. We demonstrate that $V$. parvula alters the competitive outcome during interspecies competition between $S$. mutans and S. gordonii. Consistent with this result, we show that $S$. mutans exhibits a vastly different transcriptional responses in the presence of $V$. parvula and S. gordonii.

\section{METHODS}

Bacterial strains and growth conditions. S. mutans strain UA140, S. gordonii Challis and V. parvula PK1910 were used in this study. Strain UA140 was a clinical isolate from a severe caries lesion. V. parvula strain PK1910 was formerly named Veillonella atypica PK1910 or Veillonella spp. PK1910 (Chalmers et al., 2008; Hughes et al., 1992), and is now renamed V. parvula PK1910 based upon our recent sequence analysis using the $r p o B$ gene (Qi \& Ferretti, 2011). Streptococcal species were routinely cultured in brain heart infusion (BHI) medium (Difco) or on BHI plates. V. parvula PK1910 was grown in BHI or Todd-Hewitt (TH) medium containing $0.6 \%$ sodium lactate (J. T. Baker). All cultures were grown either anaerobically ( $85 \%$ nitrogen, $5 \%$ carbon dioxide, $10 \%$ hydrogen) or aerobically ( $5 \%$ carbon dioxide) at $37{ }^{\circ} \mathrm{C}$ as static cultures.

Competition assay. Overnight, monocultures of all three species were centrifuged to remove the supernatants, and the cell pellets were resuspended in fresh $\mathrm{TH}$ broth. The cell suspension was adjusted to $\mathrm{OD}_{600}$ 0.5, and mixed in specified ratios of $S$. mutans: $S$. gordonii, $S$. mutans: V. parvula and S. mutans: S. gordonii: $V$. parvula. The initial S. mutans concentration was set at $\sim 10^{7}$ c.f.u. $\mathrm{ml}^{-1}$ in all cultures. Mono-species, dual-species or triple-species cultures were then incubated aerobically as $10 \mathrm{ml}$ static cultures in a $15 \mathrm{ml}$ culture tube in the presence of $5 \% \mathrm{CO}_{2}$. Cell growth was monitored by plating. $S$. mutans was counted by plating on BHI plates with $1 \mathrm{mg}$ spectinomycin ml $\mathrm{m}^{-1}$ (a spectinomycin-resistance gene was inserted in the chromosome of the UA140 strain), and S. gordonii was counted by plating on non-selective BHI plates, as its colonies are easily distinguished from those of $S$. mutans. V. parvula was counted by plating on BHI plates supplemented with $0.6 \%$ lactate and $12 \mu \mathrm{g}$ tetracycline $\mathrm{ml}^{-1}$. Growth curves were calculated based upon c.f.u. $\mathrm{ml}^{-1}$, and the results were the mean of at least three experiments performed on different days.

\section{Transcriptome analysis}

Cell preparation. Mono- and mixed-species cultures were grown similarly to those in the competition assay. To harvest cells for the microarray, cell growth was monitored by measuring the $\mathrm{OD}_{600}$ of the $S$. mutans monoculture. When the monoculture reached $\mathrm{OD}_{600} 0.8$ (late-exponential phase), cells were harvested by centrifugation and frozen at $-80{ }^{\circ} \mathrm{C}$ until use.

RNA extraction and microarray. RNA isolation and microarray production were performed essentially as described previously (Wu et al., 2010a), with the following addition. To ensure that equal amounts of $S$. mutans-specific RNA were used for all samples (monoand mixed-species), real-time RT-PCR was used to quantify the $S$. mutans 16S rRNA in mixed-species samples using primers described previously (Wu et al., 2010b) and the proportion of S. mutans-specific RNA was calculated.

Data analysis. Analysis of signal intensities was performed using the GeneChip operating system software (GCOS) version 1.4, and gene expression data were compared using the GCOS batch analysis function. Normalization procedures were performed directly by the software using a script designed by Affymetrix and provided with the S. mutans custom array. In addition to the general analysis required by the GeneChip, measures were also taken to address the specific issues associated with the mixed-culture microarray. For example, to assess the extent of cross-hybridization between S. mutans and $S$. gordonii and $V$. parvula, chromosomal DNA from the last two species was labelled and hybridized to the S. mutans GeneChip. Only rRNA, ribosome protein genes and some tRNA genes cross-hybridized with the $S$. mutans chip, especially for S. gordonii, and therefore these genes were eliminated from analysis. Additionally, after the initial data analysis with GCOS, the data were further normalized manually using the following steps: (1) the ratio of mixed: single species expression value for each gene was calculated with Microsoft Excel; (2) a mean ratio for each gene was calculated from four datasets; (3) a $t$ test was used to calculate the $P$ value of the fold-change for each gene using 
the gyrA gene as reference. After this normalization, a cut-off of a twofold or greater change with a $P$ value of $\leqslant 0.05$ was used to generate a dataset of genes whose expression was altered in the mixedspecies cultures as opposed to the single-species culture.

Real-time RT-PCR. Real-time RT-PCR was performed to validate the results generated by the microarray analysis. From the final list of twofold or greater responding genes, seven were randomly chosen to be further analysed. These seven genes represented a range of transcriptional responses as well as different functional groups. Primers were designed using Applied Biosystems Primer Express 3.0 software, which scans DNA sequences for primers suitable for comparative threshold value $\left(\Delta \Delta C_{\mathrm{T}}\right)$ analysis. Cells were cultured under the same conditions as described for the microarray. A $300 \mathrm{ng}$ sample of total RNA was used for the analysis, essentially as described previously ( $\mathrm{Wu}$ et al., 2010a). The gyrA gene was used as the housekeeping gene reference, and all samples included a no-reversetranscriptase control to assess genomic DNA contamination in the reactions.

\section{RESULTS AND DISCUSSION}

\section{V. parvula increases the fitness of $S$. mutans in the mixed-species culture}

As demonstrated in our previous studies (Kreth et al., 2005b; Tong et al., 2007), the mitis streptococci (i.e. S. gordonii, S. sanguinis, Streptococcus Oligofermentans, etc.) have the ability to severely inhibit $S$. mutans growth by producing large amounts of $\mathrm{H}_{2} \mathrm{O}_{2}$. However, $S$. mutans is able to inhibit the mitis streptococci by producing bacteriocins. In vitro, the outcome of this inter-species competition depends on the environmental conditions and which species occupies the niche first (Kreth et al., 2005b). Interestingly, in vivo, both $S$. mutans and the mitis streptococci can co-exist in the same biofilm community. We hypothesized that the presence of other species, such as veillonellae, might help to diffuse the conflict between the two competing groups of bacteria, resulting in their coexistence. To test this, we mixed monospecies cultures of $S$. mutans, $S$. gordonii and $V$. parvula at a ratio reminiscent of the early in vivo biofilm $(\sim 2 \%$ S. mutans, $10 \% \mathrm{~V}$. parvula and $88 \%$ S. gordonii) (Mager et al., 2003; Nyvad \& Kilian, 1990; Rosan \& Lamont, 2000). The mixed cultures were incubated as static cultures under aerobic conditions in the presence of $5 \% \mathrm{CO}_{2}$, a condition that mimics the oral cavity environment. Samples were taken every $2 \mathrm{~h}$ for viable cell counts. As shown in Fig. 1, in the S. mutans-S. gordonii dual-species culture, the growth of $S$. mutans was severely inhibited, as the growth rate and final biomass were reduced by 35 and $85 \%$, respectively. Interestingly, when $V$. parvula was introduced into the dual-species culture, the growth of $S$. mutans was rescued, resulting in a growth rate and final biomass similar to those of the $S$. mutans monoculture. This result suggested that $V$. parvula could alter the outcome of the competition between $S$. mutans and $S$. gordonii. It is important to note that in binary cultures with $S$. mutans, both $S$. gordonii and $V$. parvula grew as well as in their respective monocultures, while in tri-species cultures, S. gordonii achieved higher

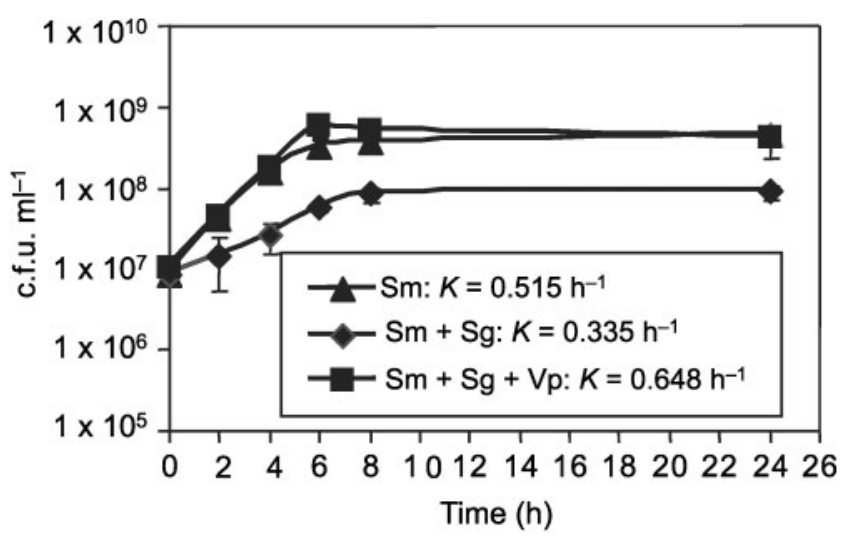

Fig. 1. Growth of $S$. mutans $(\mathrm{Sm})$ in dual-species culture with $S$. gordonii $(\mathrm{Sg})$ and in tri-species cultures with V. parvula (Vp). The experimental setup is described in Methods. Each experiment was repeated at least three times in triplicate. Presented is a typical result. $K$ is the growth rate, calculated using the formula $K=\left(\ln N-\ln N_{0}\right) / t-t_{0}$, where $N$ is the c.f.u. $\mathrm{ml}^{-1}$ at time $t$, and $N_{0}$ is the c.f.u. $\mathrm{ml}^{-1}$ at time $t_{0}$.

yields than in mono-species and dual-species cultures with S. mutans (data not shown).

\section{S. mutans responds differentially to $V$. parvula and S. gordonii}

To further explore the mechanism of this interesting interspecies interaction, microarray studies were performed using $S$. mutans monocultures $(\mathrm{Sm}), S$. mutans $-S$. gordonii dual cultures $(\mathrm{Sm}+\mathrm{Sg}), S$. mutans $-V$. parvula dual cultures $(\mathrm{Sm}+\mathrm{Vp})$ and $S$. mutans-S. gordonii-V. parvula tricultures $(\mathrm{Sm}+\mathrm{Sg}+\mathrm{Vp})$. The $S$. mutans response to coculturing with $S$. gordonii and $V$. parvula was dramatically different. When a cut-off of at least twofold with a $P$ value of $\leqslant 0.05$ was taken, a total of 400 genes were affected by coculturing with $S$. gordonii (Supplementary Table S1 and Table 2). In contrast, only 54 genes were differentially expressed by growth with $V$. parvula. In the $S$. mutans $-S$. gordonii-V. parvula tri-species culture, the total number of affected genes (354 total) was more similar to that of the $S$. mutans-S. gordonii culture. No gene was shared between the $\mathrm{Sm}+\mathrm{Sg}$ and the $\mathrm{Sm}+\mathrm{Vp}$ mixed cultures, indicating a completely different response of $S$. mutans toward the other two species (Fig. 2). In contrast, among the 54 genes affected in the $\mathrm{Sm}+\mathrm{Vp}$ cultures, 15 were shared with the tri-species culture, suggesting that $V$. parvula could still influence $S$. mutans gene expression in a similar manner, even when S. gordonii was present. Additionally, 22 genes were similarly affected in all mixed cultures, indicating a non-specific response (Fig. 2). Interestingly, more genes $(63.5 \%)$ were downregulated in the $\mathrm{Sm}+\mathrm{Sg}$ culture than in the $\mathrm{Sm}+\mathrm{Vp}$ culture $(51.8 \%)$, and about one-third of the downregulated genes in the $\mathrm{Sm}+\mathrm{Sg}$ culture were those involved in energy metabolism and biosynthesis. In 


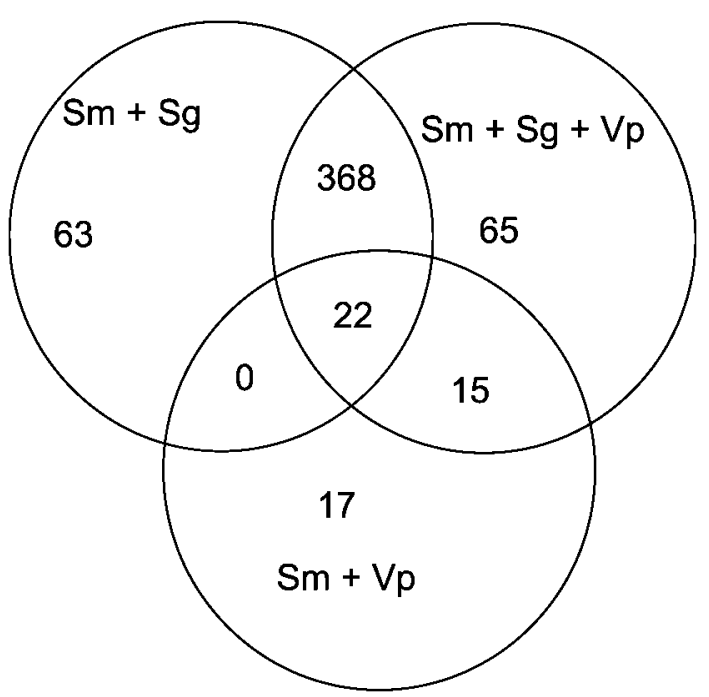

Fig. 2. Venn diagram of $S$. mutans genes affected in the three mixed-species cultures. Numbers were calculated based on genes listed in Supplementary Table S1. Only genes that were affected similarly (in the same trend) were treated as shared; those that were affected in opposite directions were treated as unique or not shared. Abbreviations: Sm, S. mutans; Sg, S. gordonii; Vp, V. parvula.

contrast, $<20 \%$ of the downregulated genes in the $\mathrm{Sm}+\mathrm{Vp}$ mixed culture belonged to this functional class.

To confirm the microarray results, real-time RT-PCR was used to measure the expression of seven genes, SMU.152, SMU.308, SMU.423, SMU.910, SMU.932, SMU.961 and SMU.1946, randomly chosen from different functional groups with different levels of response. Most of these genes exhibited the same trend of response as in the microarray (Table 3), especially for the Sm $+\mathrm{Sg}$ and the trispecies cultures, suggesting that the microarray results were likely to be an accurate reflection of the gene responses of $S$. mutans to growth in mixed cultures. Taken together, these results suggested an overall reprogramming of gene expression in S. mutans when co-cultured with S. gordonii, consistent with the observed growth inhibition of $S$. mutans by $S$. gordonii (Fig. 1). In contrast, $V$. parvula had little effect on either the growth or the gene expression of $S$. mutans in dual-species cultures; however, in trispecies culture, it had a significant effect on the gene expression pattern of $S$. mutans, which probably accounted for the observed growth rescue of the latter.

\section{The most downregulated genes in co-cultures with S. gordonii}

The most conspicuous downregulated genes encoded sugar transporters and their related metabolic enzymes. In fact, all sugar transporters, except one (SMU.2047c, EII ${ }^{\text {glu }}$ ), known to be present in the S. mutans genome (Ajdic et al., 2002; Ajdić \& Pham, 2007), were downregulated.
SMU.1957c-1961c encode fructose/mannose EII subunits A-D (Ajdić \& Pham, 2007), and are located in the same operon as SMU.1956c, a membrane protein of unknown function. In our microarray, all genes in the operon, except SMU.1958c, were downregulated two- to threefold (SMU.1958c was downregulated 1.6-fold). SMU.1841c encodes the sucrose phosphotransferase subunits EIIABC, while SMU.1843 and 1844, encode a sucrose-6-phosphate hydrolase and sucrose operon regulator, respectively. All three genes were downregulated three- to fivefold. SMU.1490c-1498c encode genes for lactose transport and metabolism. Although SMU.1490c (phospho- $\beta$-D-galactosidase) did not meet the cut-off (twofold change with a $P$ value of $<0.05$ ), all other genes in the operon were downregulated 2.5- to threefold. In addition, genes for the transport or metabolism of minor sugars and sugar alcohols were also downregulated, such as those for ribose (SMU.2142c), maltose (SMU.1568-1571), cellobiose (SMU.1596c-1600c), ribulose (SMU.270-272), sorbitol (SMU.308-314) and mannitol (SMU.1182c-1185c). Taken together, these results demonstrated that in the mixed culture with S. gordonii, S. mutans energy metabolism was suppressed. Consequently, genes involved in macromolecule biosynthesis and cell division were also downregulated (Table 1), resulting in a slower growth rate and a lower cell mass, as shown in Fig. 1.

One possibility is that the downregulation of sugar transporters and energy metabolism genes by $S$. gordonii is due to carbohydrate limitation in the mixed culture, as $S$. gordonii was numerically dominant over S. mutans $(44: 1)$. It is conceivable that this numerical advantage may have simply given them an advantage in consuming the easily metabolizable carbohydrates. The data from the tri-species culture suggested that this might be the case for the three major sugars, fructose, lactose and sucrose, while a different mechanism might explain the effect on the transport and utilization of the minor sugars and sugar alcohols (see discussion below of the genes uniquely affected in the tri-species cultures). For example, the downregulation of sugar transporters and energy metabolism genes could be due to feedback inhibition as a result of reduced glycolytic rates, similar to the feedback inhibition of growth by carbohydrate starvation observed in Lactococcus lactis (Ganesan et al., 2007). Since S. gordonii produces $\mathrm{H}_{2} \mathrm{O}_{2}$, this may inhibit $S$. mutans growth by poisoning the glycolysis system [i.e. inhibition of glyceraldehyde-3-phosphate dehydrogenase (Baldeck \& Marquis, 2008)]. This would result in accumulation of intermediates and a reduction in ATP synthesis due to the diminished downstream activities of the glycolysis pathway. Presumably, the accumulation of intermediates could also affect the expression of various metabolic genes through feedback inhibition. With a decrease in energy production, we would expect a concomitant decrease in the growth rate and final yield of $S$. mutans, as shown in Fig. 1. Accordingly, genes related to biosynthesis and cell division were downregulated as well (Table 1). Despite the 
Table 1. Differentially regulated genes discussed in the text

Abbreviations: mg, S. mutans + S. gordonii mixed culture; m, S. mutans single-species culture; mv, S. mutans + V. parvula mixed culture; mvg, S. mutans $+V$. parvula + S. gordonii mixed culture.

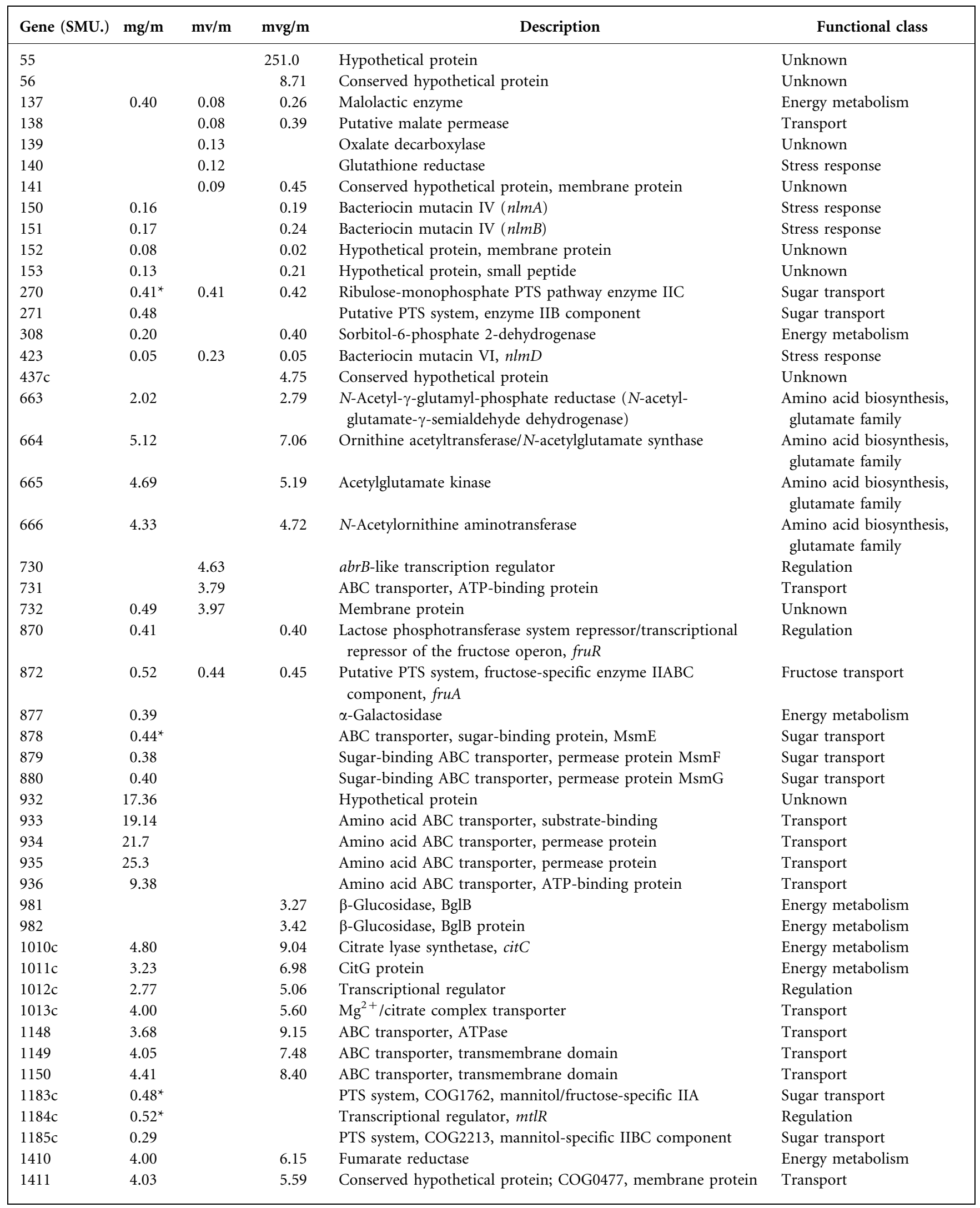


Table 1. cont.

\begin{tabular}{|c|c|c|c|c|c|}
\hline Gene (SMU.) & $\mathrm{mg} / \mathrm{m}$ & $\mathbf{m v} / \mathbf{m}$ & $\mathbf{m v g} / \mathbf{m}$ & Description & Functional class \\
\hline $1492 \mathrm{c}$ & 0.39 & & $0.53^{*}$ & PTS system, cellobiose-specific IIA component & Sugar transport \\
\hline $1493 c$ & 0.43 & & & Tagatose-1,6-bisphosphate aldolase & Energy metabolism \\
\hline $1495 \mathrm{c}$ & 0.31 & & 0.39 & Galactose-6-phosphate isomerase, LacB & Energy metabolism \\
\hline $1496 c$ & 0.32 & & & Galactose-6-phosphate isomerase, LacA & Energy metabolism \\
\hline $1498 \mathrm{c}$ & 0.34 & & 0.47 & Lactose phosphotransferase system repressor, LacR & Energy metabolism \\
\hline 1519 & 3.86 & & 3.45 & Putative amino acid ABC transporter, ATP-binding protein & Transport \\
\hline 1520 & 3.55 & & $3.13^{*}$ & Putative $\mathrm{ABC}$ transporter, glutamate-binding protein & Transport \\
\hline 1521 & 2.86 & & 2.17 & Glutamate $\mathrm{ABC}$ transporter, permease & Glutamate transport \\
\hline 1522 & 2.15 & & 1.73 & Glutamate $\mathrm{ABC}$ transporter, permease & Glutamate transport \\
\hline 1568 & 0.10 & & 0.32 & Maltose maltodextrin-binding protein MalE & Sugar transport \\
\hline 1569 & 0.19 & & 0.39 & Maltodextrin $\mathrm{ABC}$ transporter, permease protein $\mathrm{MalF}$ & Sugar transport \\
\hline 1570 & 0.22 & & 0.33 & Maltose maltodextrin $\mathrm{ABC}$ transporter, $\mathrm{MalG}$ permease & Sugar transport \\
\hline 1571 & 0.17 & & 0.30 & Maltose $\mathrm{ABC}$ transporter, ATP-binding protein, MsmK & Sugar transport \\
\hline $1574 \mathrm{c}$ & & & 4.03 & Conserved hypothetical protein & Unknown \\
\hline $1597 \mathrm{c}$ & 0.31 & & & Conserved hypothetical protein, membrane protein & Unknown \\
\hline $1598 c$ & $0.35^{*}$ & & & Putative PTS system, cellobiose-specific IIA component, CelC & Cellobiose uptake \\
\hline $1599 \mathrm{c}$ & $0.35^{\star}$ & & & Transcriptional regulator, CelR & Regulation \\
\hline $1600 \mathrm{c}$ & 0.12 & & 0.27 & PTS system, cellobiose-specific IIB component, CelB & Cellobiose uptake \\
\hline $1818 \mathrm{c}$ & & & 7.86 & Hypothetical protein & Unknown \\
\hline $1841 \mathrm{c}$ & 0.30 & & 0.46 & PTS system, sucrose-specific IIABC component & Sugar transport \\
\hline 1843 & 0.16 & & 0.23 & Sucrose-6-phosphate hydrolase & Energy metabolism \\
\hline 1844 & 0.26 & & 0.38 & Sucrose operon repressor & Regulation \\
\hline 1897 & 4.42 & & 2.50 & $\mathrm{ABC}$ transporter, $\mathrm{ATP}$-binding protein; similar to $\mathrm{BlpA}$ & Transport \\
\hline 1898 & 3.05 & & 3.91 & $\mathrm{ABC}$ transporter, $\mathrm{ATP}$-binding and permease element & Transport \\
\hline 1899 & 4.17 & & 4.90 & $\mathrm{ABC}$ transporter, ATP-binding and permease protein & Transport \\
\hline 1900 & 4.32 & & 5.81 & $\mathrm{ABC}$ transporter & Transport \\
\hline $1902 \mathrm{c}$ & 0.40 & & 0.40 & Bacteriocin-like peptide & Unknown \\
\hline $1903 c$ & 0.18 & 0.34 & 0.20 & Bacteriocin-like peptide & Unknown \\
\hline $1904 c$ & 0.25 & $0.53^{\star}$ & 0.32 & Possible bacteriocin immunity protein & Unknown \\
\hline $1905 c$ & 0.21 & 0.49 & 0.29 & CSP-regulated bacteriocin-like peptide & Unknown \\
\hline $1906 \mathrm{c}$ & 0.12 & 0.25 & 0.08 & CSP-regulated bacteriocin-like peptide & Unknown \\
\hline $1956 c$ & 0.32 & & 0.41 & Hypothetical protein, membrane protein & Unknown \\
\hline $1957 c$ & 0.47 & & 0.58 & Fructose-specific enzyme IID component & Sugar transport \\
\hline $1960 c$ & 0.40 & & 0.61 & Fructose-specific enzyme IIB component & Sugar transport \\
\hline $1961 c$ & 0.29 & & 0.49 & Fructose-specific IIA component & Sugar transport \\
\hline $2142 c$ & 2.00 & & 2.15 & COG0698, ribose-5-phosphate isomerase & Energy metabolism \\
\hline
\end{tabular}

${ }^{*}$ Denotes genes with a $P$ value $\geqslant 0.05$ but $\leqslant 0.1$. These genes were included in the table only when other members of the same operon exhibited similar changes in gene expression and met the $P$ value $<0.05$ cut-off.

ubiquitous effect upon sugar transport operons, it was surprising to observe that the putative glucose transporter (SMU.2047c) was not affected. SMU.2047c has been annotated as a glucose transporter based on its homology with the EII ${ }^{\text {glc }}$ of Bacillus subtilis (Abranches et al., 2006); however, inactivation of this gene did not affect cell growth in medium containing glucose as the carbon source (Abranches et al., 2006). Our results appear to be consistent with this observation.

\section{The most important upregulated genes in co-cultures with S. gordonii}

While S. mutans growth rate was reduced in the mixed culture with $S$. gordonii, its growth persisted (Fig. 1), which suggests that $S$. mutans likely used alternative energy sources to compensate for its reduced capacity to metabolize carbohydrates. Inspection of the upregulated genes in the dataset (Table 1) may provide further evidence of this. We identified four upregulated operons involved in generating energy from alternative sources: the citrate lyase synthetase operon (SMU.1010c-1013c), the fumarate reductase operon (SMU.1410-1411), the glutamate transporter operon (SMU.1519-1522) (Krastel et al., 2010) and the glutamate family amino acid synthesis operon (SMU.663-666). All genes in these operons were upregulated three- to fivefold in the mixed culture with $S$. gordonii, although SMU.1520 had a slightly higher $P$ value (0.06). SMU.1010c encodes citrate lyase synthetase. Citrate lyase catalyses the cleavage of citrate to acetate and oxaloacetate; the latter is an intermediate of the tricarboxylic acid (TCA) cycle, and can be further metabolized 
Table 2. Summary of differentially regulated genes in different functional classes

Abbreviations: Sm, S. mutans; Sg, S. gordonii; Vp, V. parvula. Down, downregulation; up, upregulation.

\begin{tabular}{|c|c|c|c|c|c|c|}
\hline \multirow[t]{2}{*}{ Functional class } & \multicolumn{2}{|c|}{$\mathrm{Sm}+\mathrm{Sg}$} & \multicolumn{2}{|c|}{$S m+V p$} & \multicolumn{2}{|c|}{$S m+S g+V p$} \\
\hline & Down & Up & Down & Up & Down & Up \\
\hline Energy metabolism & 32 & 8 & 4 & 1 & 23 & 10 \\
\hline $\begin{array}{l}\text { Biosynthesis/central } \\
\text { metabolism }\end{array}$ & 45 & 27 & 1 & 4 & 37 & 18 \\
\hline Cell division & 10 & 3 & 0 & 2 & 5 & 5 \\
\hline Sugar transport & 16 & 0 & 2 & 0 & 13 & 0 \\
\hline Transport & 9 & 32 & 4 & 3 & 11 & 30 \\
\hline Regulation & 31 & 5 & 1 & 4 & 25 & 12 \\
\hline Stress response & 14 & 7 & 2 & 2 & 14 & 8 \\
\hline Adhesion & 1 & 4 & 1 & 2 & 0 & 4 \\
\hline Unassigned & 10 & 6 & 0 & 2 & 6 & 9 \\
\hline Unknown & 84 & 56 & 12 & 7 & 69 & 69 \\
\hline Total & 252 & 148 & 28 & 26 & 203 & 161 \\
\hline
\end{tabular}

to enter into the pyruvate metabolic pathway of the glycolysis cycle or for amino acid synthesis (Korithoski et al., 2005) (Fig. 3). Citrate lyase is post-translationally activated, and its activation requires citrate lyase synthetase and the CitG protein (encoded by SMU.1011c) (Schneider et al., 2000). SMU.1012c encodes a transcription regulator, which is probably responsible for the regulation of the operon, and SMU.1013c encodes a $\mathrm{Mg}^{2+}$ /citrate complex transporter. Thus, by increasing citrate transport and activating citrate lyase activity, $S$. mutans could use citrate as an alternative energy source. In lactic acid bacteria, citrate has been used to generate proton motive force and to increase acid tolerance (Garcia-Quintáns et al., 1998; Ramos et al., 1994, 1995). In S. mutans, citrate has been shown to be transported and metabolized, though not as a principal carbon source (Korithoski et al., 2005). Moreover, preincubation with citrate increases S. mutans survival at acidic $\mathrm{pH}$ (Korithoski et al., 2005). Our results suggested that citrate transport and metabolism may play an important role in S. mutans persistence in the early biofilm, where the mitis streptococci are numerically dominant (Rosan \& Lamont, 2000).

The possible function of glutamate transport (SMU.15191522 ) and glutamate family biosynthesis genes in S. mutans survival is illustrated in Fig. 3. After glutamate is transported into the cell by the gene products of SMU.15191522 , it could be acetylated by SMU.664 to $N$-acetyl glutamate, which is then phosphorylated by SMU.665 to form $\mathrm{N}$-acetyl glutamate phosphate. $\mathrm{N}$-acetyl glutamate phosphate is then reduced by the gene product of SMU.663 to $\mathrm{N}$-acetyl glutamate phosphate semialdehyde, which is converted to $\mathrm{N}$-acetyl ornithine by the gene product of SMU.666. Acetyl ornithine can enter the urea cycle, through which it is converted into arginine and fumarate. Arginine can be used directly for protein synthesis, while fumarate can be converted to succinate by fumarate reductase, the gene product of SMU.1610. Succinate is an intermediate of the TCA cycle, through which intermediate metabolites and ATP can be generated. Although S. mutans does not have a complete TCA cycle, a partial TCA cycle is present (Ajdić et al., 2002). Interestingly, glutamate has also been found to play an important role in regulating the activity of citrate lyase in Clostridium sphenoides (Antranikian \& Gottschalk, 1989). Although the effect of glutamate on citrate lyase activity in S. mutans is not known, a recent study found that the glutamate transport operon plays an important role in acid tolerance of $S$. mutans (Krastel et al., 2010), suggesting a possible link with citrate metabolism (Korithoski et al., 2005). Thus, these four upregulated operons may actually form a network linking the transport and metabolism of glutamate and citrate with three major metabolic cycles (the urea cycle, the TCA cycle and glycolysis) to generate energy and metabolic intermediates for $S$. mutans when sugar metabolism is suppressed (Fig. 3).

Table 3. Comparison of microarray and real-time RT-PCR results for selected genes

Abbreviations: Sm, S. mutans; Sg, S. gordonii; Vp, V. parvula.

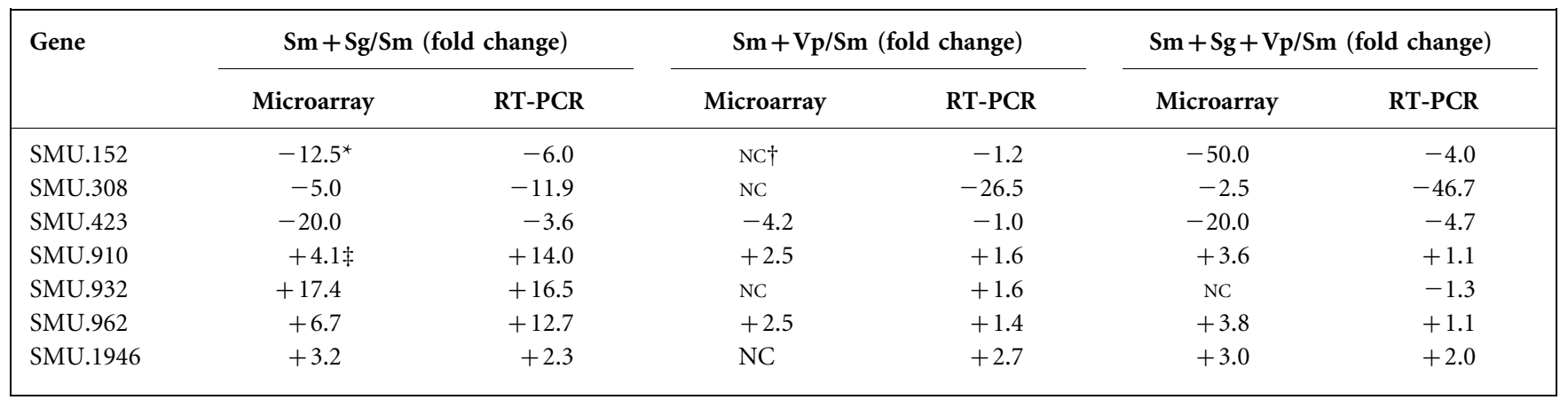

${ }^{\star}$ Minus value: downregulated in mixed culture.

$\dagger \mathrm{NC}$, No change.

\$Plus value: upregulated in mixed culture. 


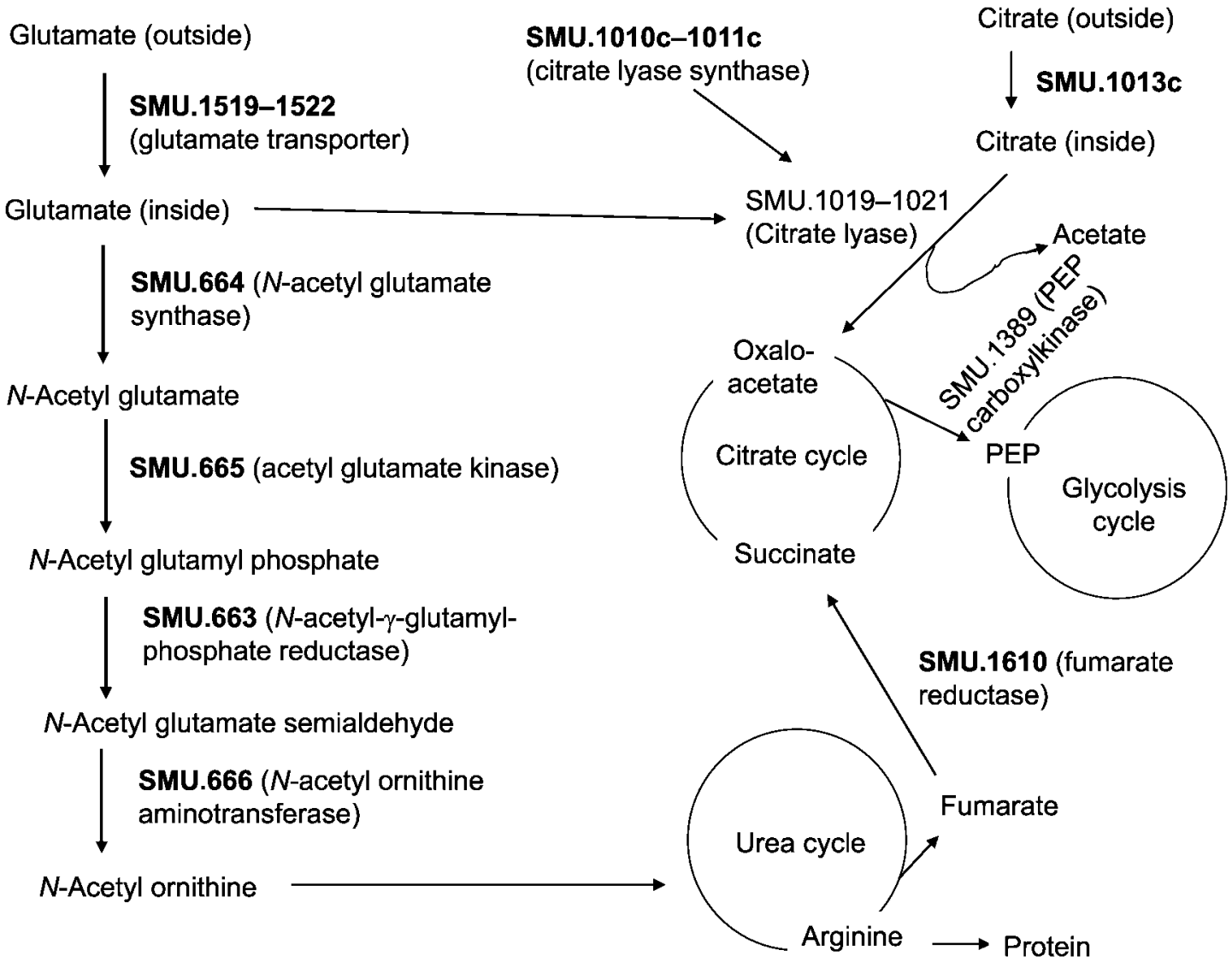

Fig. 3. Proposed pathways upregulated in the $S$. mutans + S. gordonii mixed cultures. Genes in bold type are upregulated in the mixed culture; genes in plain type exist in the $S$. mutans genome, but were not upregulated in the microarray. The pathways are proposed based on the Kyoto Encyclopedia of Genes and Genomes (KEGG) pathway database (http://www.genome.jp/kegg/ pathway.html\#energy).

Another set of significantly upregulated genes was the cysteine transport operon (SMU.932-936) (Sperandio et al., 2010), which was upregulated 10-25 fold (Table 1). Cysteine is an essential nutrient for $S$. mutans growth (Terleckyj \& Shockman, 1975). Cysteine can be converted to methionine by cystathionine $\gamma$-synthase (SMU.1675) and homocysteine $S$-methyltransferase (SMU.873 and 874). It can also be used in a thiol form for S. mutans $\mathrm{H}_{2} \mathrm{O}_{2}$ resistance (Thomas et al., 1983). In our case, we propose that cysteine is more likely to be used for the latter purpose, as $\mathrm{H}_{2} \mathrm{O}_{2}$ production by $S$. gordonii (or S. sanguinis) has been shown to be the primary inhibitor of $S$. mutans growth in mixed cultures (Kreth et al., 2005b). In addition to the cysteine transporter, two more operons encoding $\mathrm{ABC}$ transporters were also upregulated (SMU.1148-1150, SMU.1897-1900). Interestingly, SMU.1897-1900 have been shown to encode the transporter for the secretion of CSP (Hale et al., 2005), while SMU.1148-1150 exhibit homology to genes of lantibiotic peptide $\mathrm{ABC}$ transporters. In our previous study, we have shown that CSP controls the expression of a group of bacteriocin genes whose products specifically kill the mitis streptococci, such as S. gordonii, to cause DNA release for uptake by competent $S$. mutans cells (Kreth et al., 2005a). Wang \& Kuramitsu (2005) demonstrated that to fight back, S. gordonii produces a protease that degrades CSP, thus downregulating bacteriocin production genes in $S$. mutans. Our results here suggest that $S$. mutans may further counter this strategy by increasing CSP export.

\section{The most affected genes in co-cultures with $V$. parvula}

As shown in Table 1, few genes were affected in the $\mathrm{Sm}+\mathrm{Vp}$ mixed culture more than threefold. One operon that stands out as the most downregulated encodes genes for malate transport and metabolism (SMU.137-141). All genes in this operon were downregulated $\sim 10$-fold. Malolactic fermentation (MLF) is a form of secondary metabolism carried out by lactic acid bacteria, including $S$. mutans, to produce ATP. The dicarboxylic acid L-malate is converted to the monocarboxylic acid lactate and $\mathrm{CO}_{2}$ by the malolactic enzyme (encoded by SMU.137). Since malic acid is a stronger acid than lactic acid, this reaction creates 
a $\mathrm{pH}$ gradient between the cytoplasm and the outside environment, which drives ATP synthesis by the $\mathrm{F}\left(\mathrm{H}^{+}\right)$ATPase (Salema et al., 1996; Sheng \& Marquis, 2007). In S. mutans it has been shown that MLF is induced by low $\mathrm{pH}$ and the presence of L-malate (Sheng \& Marquis, 2007), and accordingly the gene expression of SMU.137140 is also induced by low $\mathrm{pH}$ and the presence of malate in the growth medium (Lemme et al., 2010). In batch culture, the expression of this operon appears to follow the growth curve, with expression levels peaking at early stationary phase (Lemme et al., 2010), when the growth medium had been acidified. Since our samples for microarray analysis were taken at late-exponential phase, we suspect that the malolactic operon in the monoculture was induced by the low $\mathrm{pH}$ at this stage. Thus, the downregulation of the malolactic operon in the $\mathrm{Sm}+\mathrm{Vp}$ mixedspecies culture could be due to the higher $\mathrm{pH}$ in the mixed culture because of the consumption of lactic acid by $V$. parvula. Indeed, the $\mathrm{pH}$ of the $S$. mutans monoculture at late-exponential phase was found to be approximately 5.4, while in the mixed culture it was approximately 6.4 (unpublished data). Thus, the higher $\mathrm{pH}$ of the $\mathrm{Sm}+\mathrm{Vp}$ culture presumably resulted in lower expression of the malolactic enzyme operon.

The most significantly upregulated genes in the $\mathrm{Sm}+\mathrm{Vp}$ mixed culture were SMU.730-732, which constitute a single operon and are upregulated approximately fourfold. SMU.730 encodes a 70 aa protein with an AbrB-like transcription regulator domain. SMU.731 and 732 encode an $\mathrm{ABC}$ transporter of unknown function. At present, the function of this operon and its relationship to mixedspecies growth are unknown.

\section{Genes uniquely affected in the tri-species culture}

Despite the large overlap between the $\mathrm{Sm}+\mathrm{Sg}$ and tri-species microarray datasets (Fig. 2), we identified some important differences between them that may provide some insight into the improved growth of $S$. mutans in the tri-species culture. The most conspicuous change in gene expression pattern in the tri-species culture was the upregulation of five operons involved in sugar transport and metabolism: the raffinose transport system (SMU.877-880), the mannitol transport operon (SMU.1183c-1185c), the maltose/maltodextrin transporters (SMU.1568-1571), the cellobiose uptake system (SMU.1597c-1599c) and the $\beta$-galactosidase genes (SMU.981-982). For the raffinose transport system, the mannitol transport operon and the cellobiose uptake system, the gene expression level in the tri-species culture was the same as in the Sm monospecies culture, while in the $\mathrm{Sm}+\mathrm{Sg}$ dual-species culture, these genes were all downregulated twoto threefold (Table 1). For the maltose/maltodextrin transporters and the $\beta$-galactosidase genes, expression increased 1.6- to threefold in the tri-species culture with respect to the dual-species culture. Interestingly, genes for the transport and utilization of major sugars, such as sucrose (SMU.1841-1844), fructose/mannose (SMU.1957-1961) and lactose (SMU.1490-1498), were not upregulated in the tri-species culture. This suggested that the presence of $V$. parvula in the mixed-species culture somehow increased the preference of $S$. mutans for the minor sugars and sugar alcohols. In addition, the citrate operon (SMU.1010c1013c), which was already upregulated in the $\mathrm{Sm}+\mathrm{Sg}$ dual-species culture, was increased even further by an additional 1.5- to twofold in the tri-species culture, further boosting the capacity of $S$. mutans for utilizing alternative energy sources.

In addition to the increased expression of various sugar transporters and the citrate operon, the tri-species culture also specifically increased the expression of several hypothetical genes. For example, SMU.55 and SMU.56 were upregulated $>250$-fold and over eightfold, respectively, in the tri-species culture. In fact, these genes exhibited little or no detectable signal in both the mono- and dual-species cultures, suggesting they were uniquely expressed in the tri-species condition. SMU.55 encodes an 87 aa protein of unknown function, and SMU.56 encodes a streptococcus-specific peptide of 42 aa; both genes appear to be co-transcribed. Additionally, several genes encoding hypothetical proteins (SMU.437c, SMU.955, SMU.1574c and SMU.1818c) were uniquely upregulated more than fourfold in the tri-species culture (Table 1). Whether these genes play any role in the interspecies interaction awaits further experimentation.

\section{Genes commonly affected in all mixed-species cultures}

Among the 22 commonly affected genes in all mixed cultures, a group of bacteriocin genes are worth noting. SMU.423 (nlmD), $1905 \mathrm{c}$ and $1906 \mathrm{c}$ encode putative bacteriocins (Xie et al., 2010) and 1904c encodes a putative bacteriocin immunity/modification protein, all of which have been shown to be regulated by CSP in S. mutans (Kreth et al., 2006; van der Ploeg, 2005; Perry et al., 2009). $S$. gordonii has been shown to downregulate CSP-induced bacteriocin genes of $S$. mutans by degrading CSP with a peptidase (Wang \& Kuramitsu, 2005). This suggests that the downregulated bacteriocin-like genes could be a result of CSP degradation by $S$. gordonii in the mixed culture. Bacteriocin production is also a stress response of $S$. mutans (Kreth et al., 2005a, b; Qi et al., 2001), which suggests that the downregulation of bacteriocin gene expression in the mixed culture with $V$. parvula is an indication of no stress for $S$. mutans. In fact, S. mutans grows equally well in the spent medium of $V$. parvula as in fresh medium, and slightly better in mixed culture with $V$. parvula than in monoculture (data not shown), further supporting the notion that a mutualistic relationship exists between S. mutans and V. parvula.

\section{Ecological implications of this study}

How different species co-exist in a multispecies ecosystem is a particularly important aspect of polymicrobial disease 


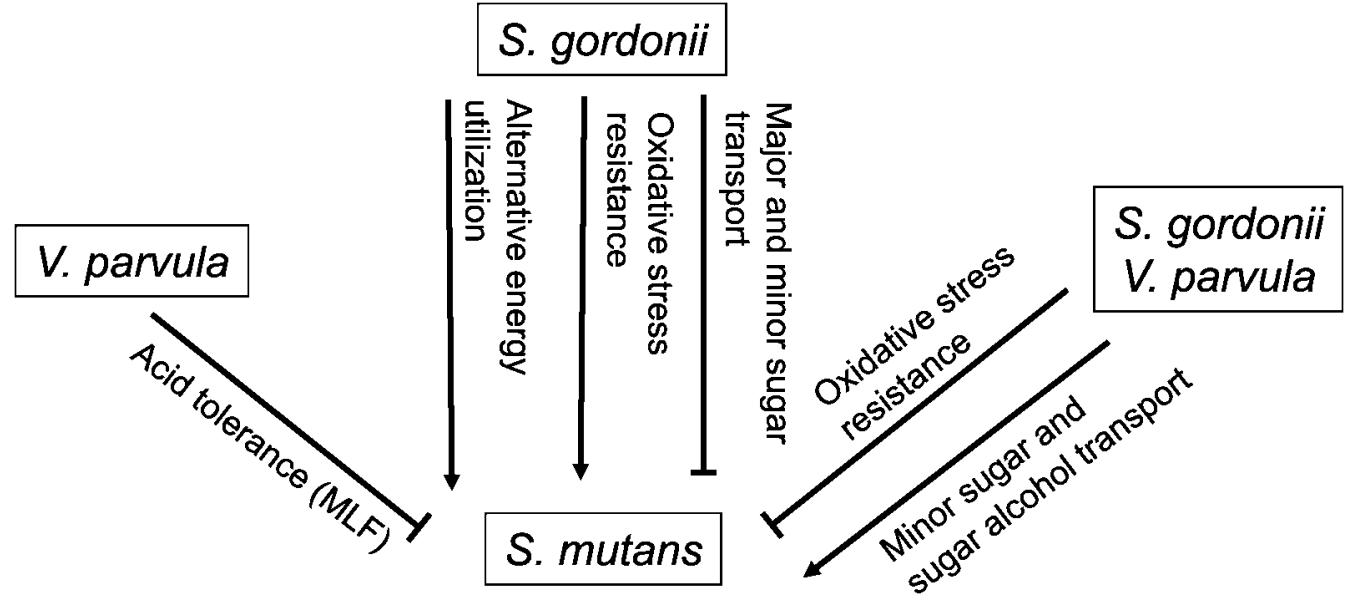

Fig. 4. Summary of S. mutans responses in mixed cultures. Blocked lines, downregulated; arrowed lines, upregulated. See text for details.

aetiology (Bakaletz, 2004; Brogden et al., 2005; Rogers et al., 2010; Sibley et al., 2006). In this study, we used threespecies mixed cultures as a facile model of the early microbial community of the supragingival plaque. Our results demonstrate that introducing a 'friendly' species into a mixed culture of competitors could affect this competition, resulting in the co-existence of all three species. Using a microarray analysis we further demonstrate that $S$. mutans responds differentially to its friend (V. parvula) and foe (S. gordonii). As illustrated in Fig. 4, when engaged in competition with S. gordonii, S. mutans sugar uptake and metabolic genes are downregulated, resulting in slower growth and a lower cell mass. To remain persistent in the mixed culture, S. mutans upregulated genes for alternative energy source acquisition and utilization, as well as genes to deal with environmental stress $\left(\mathrm{H}_{2} \mathrm{O}_{2}\right)$ in the mixed culture. Thus, in the mixed culture with $S$. gordonii, nutrition and stress appear to be the major factors affecting $S$. mutans fitness. In co-cultures with $V$. parvula, the consumption of lactic acid appears to abolish the requirement for MLF, since $S$. mutans strongly downregulated these genes. Presumably, this is due to the increased $\mathrm{pH}$ of the culture. When $V$. parvula was introduced into the mixed culture of the two competitors, $S$. mutans growth inhibition was rescued, and genes for the uptake and metabolism of minor sugars were upregulated, while genes required for $\mathrm{H}_{2} \mathrm{O}_{2}$ stress (cysteine transporters) were downregulated. Thus, $V$. parvula appeared to promote $S$. mutans growth in the tri-species culture by mitigating the two major factors that limit $S$. mutans growth in the presence of $S$. gordonii. Although the exact mechanism by which $V$. parvula promoted $S$. mutans growth needs to be further explored, our results at least provide a reasonable explanation for the numerous in vivo observations that veillonellae appear to be associated with carious lesions despite their consumption of lactic acid produced by cariogenic bacteria.

\section{ACKNOWLEDGEMENTS}

We thank Dr Zhiyuan Chen for helping with the microarray data deposition. This work was supported in part by NIH grants R15 DE019940 to F. Q. and a Centers of Biomedical Research Excellence (COBRE) P20-RR018741-05 grant to J. M.

\section{REFERENCES}

Aas, J. A., Paster, B. J., Stokes, L. N., Olsen, I. \& Dewhirst, F. E. (2005). Defining the normal bacterial flora of the oral cavity. J Clin Microbiol 43, 5721-5732.

Aas, J. A., Griffen, A. L., Dardis, S. R., Lee, A. M., Olsen, I., Dewhirst, F. E., Leys, E. J. \& Paster, B. J. (2008). Bacteria of dental caries in primary and permanent teeth in children and young adults. J Clin Microbiol 46, 1407-1417.

Abranches, J., Candella, M. M., Wen, Z. T., Baker, H. V. \& Burne, R. A. (2006). Different roles of EIIAB ${ }^{\mathrm{Man}}$ and EII ${ }^{\mathrm{Glc}}$ in regulation of energy metabolism, biofilm development, and competence in Streptococcus mutans. J Bacteriol 188, 3748-3756.

Ajdić, D. \& Pham, V. T. (2007). Global transcriptional analysis of Streptococcus mutans sugar transporters using microarrays. J Bacteriol 189, 5049-5059.

Ajdić, D., McShan, W. M., McLaughlin, R. E., Savić, G., Chang, J., Carson, M. B., Primeaux, C., Tian, R., Kenton, S. \& other authors (2002). Genome sequence of Streptococcus mutans UA159, a cariogenic dental pathogen. Proc Natl Acad Sci U S A 99, 14434-14439.

Antranikian, G. \& Gottschalk, G. (1989). Phosphorylation of citrate lyase ligase in Clostridium sphenoides and regulation of anaerobic citrate metabolism in other bacteria. Biochimie 71, 1029-1037.

Bakaletz, L. O. (2004). Developing animal models for polymicrobial diseases. Nat Rev Microbiol 2, 552-568.

Baldeck, J. D. \& Marquis, R. E. (2008). Targets for hydrogen-peroxideinduced damage to suspension and biofilm cells of Streptococcus mutans. Can J Microbiol 54, 868-875.

Becker, M. R., Paster, B. J., Leys, E. J., Moeschberger, M. L., Kenyon, S. G., Galvin, J. L., Boches, S. K., Dewhirst, F. E. \& Griffen, A. L. (2002). Molecular analysis of bacterial species associated with childhood caries. J Clin Microbiol 40, 1001-1009. 
Brogden, K. A., Guthmiller, J. M. \& Taylor, C. E. (2005). Human polymicrobial infections. Lancet 365, 253-255.

Caufield, P. W., Cutter, G. R. \& Dasanayake, A. P. (1993). Initial acquisition of mutans streptococci by infants: evidence for a discrete window of infectivity. J Dent Res 72, 37-45.

Caufield, P. W., Dasanayake, A. P., Li, Y., Pan, Y., Hsu, J. \& Hardin, J. M. (2000). Natural history of Streptococcus sanguinis in the oral cavity of infants: evidence for a discrete window of infectivity. Infect Immun 68, 4018-4023.

Chalmers, N. I., Palmer, R. J., Jr, Cisar, J. O. \& Kolenbrander, P. E. (2008). Characterization of a Streptococcus sp.-Veillonella sp. community micromanipulated from dental plaque. J Bacteriol 190, 8145-8154.

Dewhirst, F. E., Chen, T., Izard, J., Paster, B. J., Tanner, A. C., Yu, W. H., Lakshmanan, A. \& Wade, W. G. (2010). The human oral microbiome. J Bacteriol 192, 5002-5017.

Diaz, P. I., Chalmers, N. I., Rickard, A. H., Kong, C., Milburn, C. L., Palmer, R. J., Jr \& Kolenbrander, P. E. (2006). Molecular characterization of subject-specific oral microflora during initial colonization of enamel. Appl Environ Microbiol 72, 2837-2848.

Ganesan, B., Stuart, M. R. \& Weimer, B. C. (2007). Carbohydrate starvation causes a metabolically active but nonculturable state in Lactococcus lactis. Appl Environ Microbiol 73, 2498-2512.

Garcia-Quintáns, N., Magni, C., de Mendoza, D. \& López, P. (1998). The citrate transport system of Lactococcus lactis subsp. lactis biovar diacetylactis is induced by acid stress. Appl Environ Microbiol 64, 850857.

Hale, J. D., Heng, N. C., Jack, R. W. \& Tagg, J. R. (2005). Identification of $n l m T E$, the locus encoding the $\mathrm{ABC}$ transport system required for export of nonlantibiotic mutacins in Streptococcus mutans. J Bacteriol 187, 5036-5039.

Hughes, C. V., Kolenbrander, P. E., Andersen, R. N. \& Moore, L. V. (1988). Coaggregation properties of human oral Veillonella spp.: relationship to colonization site and oral ecology. Appl Environ Microbiol 54, 1957-1963.

Hughes, C. V., Andersen, R. N. \& Kolenbrander, P. E. (1992). Characterization of Veillonella atypica PK1910 adhesin-mediated coaggregation with oral Streptococcus spp. Infect Immun 60, 1178-1186.

Keijser, B. J., Zaura, E., Huse, S. M., van der Vossen, J. M., Schuren, F. H., Montijn, R. C., ten Cate, J. M. \& Crielaard, W. (2008). Pyrosequencing analysis of the oral microflora of healthy adults. J Dent Res 87, 1016-1020.

Kolenbrander, P. E., Palmer, R. J., Jr, Rickard, A. H., Jakubovics, N. S., Chalmers, N. I. \& Diaz, P. I. (2006). Bacterial interactions and successions during plaque development. Periodontol 2000 42, 47-79.

Kolenbrander, P. E., Palmer, R. J., Jr, Periasamy, S. \& Jakubovics, N. S. (2010). Oral multispecies biofilm development and the key role of cell-cell distance. Nat Rev Microbiol 8, 471-480.

Korithoski, B., Krastel, K. \& Cvitkovitch, D. G. (2005). Transport and metabolism of citrate by Streptococcus mutans. J Bacteriol 187, 44514456.

Krastel, K., Senadheera, D. B., Mair, R., Downey, J. S., Goodman, S. D. \& Cvitkovitch, D. G. (2010). Characterization of a glutamate transporter operon, glnQHMP, in Streptococcus mutans and its role in acid tolerance. J Bacteriol 192, 984-993.

Kreth, J., Merritt, J., Shi, W. \& Qi, F. (2005a). Co-ordinated bacteriocin production and competence development: a possible mechanism for taking up DNA from neighbouring species. Mol Microbiol 57, 392-404.

Kreth, J., Merritt, J., Shi, W. \& Qi, F. (2005b). Competition and coexistence between Streptococcus mutans and Streptococcus sanguinis in the dental biofilm. J Bacteriol 187, 7193-7203.
Kreth, J., Merritt, J., Zhu, L., Shi, W. \& Qi, F. (2006). Cell density- and ComE-dependent expression of a group of mutacin and mutacin-like genes in Streptococcus mutans. FEMS Microbiol Lett 265, 11-17.

Lemme, A., Sztajer, H. \& Wagner-Döbler, I. (2010). Characterization of $m l e R$, a positive regulator of malolactic fermentation and part of the acid tolerance response inStreptococcus mutans. BMC Microbiol 10,58 .

Loesche, W. J. (1986). Role of Streptococcus mutans in human dental decay. Microbiol Rev 50, 353-380.

Luppens, S. B., Kara, D., Bandounas, L., Jonker, M. J., Wittink, F. R., Bruning, O., Breit, T. M., Ten Cate, J. M. \& Crielaard, W. (2008). Effect of Veillonella parvula on the antimicrobial resistance and gene expression of Streptococcus mutans grown in a dual-species biofilm. Oral Microbiol Immunol 23, 183-189.

Mager, D. L., Ximenez-Fyvie, L. A., Haffajee, A. D. \& Socransky, S. S. (2003). Distribution of selected bacterial species on intraoral surfaces. J Clin Periodontol 30, 644-654.

Marsh, P. D. (1994). Microbial ecology of dental plaque and its significance in health and disease. Adv Dent Res 8, 263-271.

Marsh, P. D. (1999). Microbiologic aspects of dental plaque and dental caries. Dent Clin North Am 43, 599-614, v-vi.

Marsh, P. D. (2006). Dental diseases - are these examples of ecological catastrophes? Int J Dent Hyg 4 (Suppl. 1), 3-10, discussion 50-52.

Mikx, F. H. \& Van der Hoeven, J. S. (1975). Symbiosis of Streptococcus mutans and Veillonella alcalescens in mixed continuous cultures. Arch Oral Biol 20, 407-410.

Mikx, F. H., van der Hoeven, J. S., König, K. G., Plasschaert, A. J. \& Guggenheim, B. (1972). Establishment of defined microbial ecosystems in germ-free rats. I. The effect of the interactions of Streptococcus mutans or Streptococcus sanguis with Veillonella alcalescens on plaque formation and caries activity. Caries Res 6, 211-223.

Mikx, F. H., van der Hoeven, J. S., Plasschaert, A. J. \& König, K. G. (1976). Establishment and symbiosis of Actinomyces viscosus, Streptococcus sanguis and Streptococcus mutans in germ-free OsborneMendel rats. Caries Res 10, 123-132.

Nyvad, B. \& Kilian, M. (1990). Microflora associated with experimental root surface caries in humans. Infect Immun 58, 1628-1633.

Palmer, R. J., Jr, Diaz, P. I. \& Kolenbrander, P. E. (2006). Rapid succession within the Veillonella population of a developing human oral biofilm in situ. J Bacteriol 188, 4117-4124.

Paster, B. J., Boches, S. K., Galvin, J. L., Ericson, R. E., Lau, C. N., Levanos, V. A., Sahasrabudhe, A. \& Dewhirst, F. E. (2001). Bacterial diversity in human subgingival plaque. J Bacteriol 183, 3770-3783.

Paster, B. J., Olsen, I., Aas, J. A. \& Dewhirst, F. E. (2006). The breadth of bacterial diversity in the human periodontal pocket and other oral sites. Periodontol 2000 42, 80-87.

Perry, J. A., Jones, M. B., Peterson, S. N., Cvitkovitch, D. G. \& Lévesque, C. M. (2009). Peptide alarmone signalling triggers an autoactive bacteriocin necessary for genetic competence. Mol Microbiol 72, 905-917.

Qi, F. \& Ferretti, J. (2011). The Streptococcus-Veillonella community: how genome sequencing aids our understanding of interspecies interaction. In Oral Microbial Communities: Genomic Inquiry and Interspecies Communication, pp. 351-370. Edited by P. E. Kolenbrander. Washington, DC: American Society for Microbiology.

Qi, F., Chen, P. \& Caufield, P. W. (2001). The group I strain of Streptococcus mutans, UA140, produces both the lantibiotic mutacin I and a nonlantibiotic bacteriocin, mutacin IV. Appl Environ Microbiol 67, 15-21. 
Ramos, A., Poolman, B., Santos, H., Lolkema, J. S. \& Konings, W. N. (1994). Uniport of anionic citrate and proton consumption in citrate metabolism generates a proton motive force in Leuconostoc oenos. J Bacteriol 176, 4899-4905.

Ramos, A., Lolkema, J. S., Konings, W. N. \& Santos, H. (1995). Enzyme basis for $\mathrm{pH}$ regulation of citrate and pyruvate metabolism by Leuconostoc oenos. Appl Environ Microbiol 61, 1303-1310.

Rogers, G. B., Hoffman, L. R., Whiteley, M., Daniels, T. W., Carroll, M. P. \& Bruce, K. D. (2010). Revealing the dynamics of polymicrobial infections: implications for antibiotic therapy. Trends Microbiol 18, 357-364.

Rogosa, M. \& Bishop, F. S. (1964). The genus Veillonella. II. Nutritional studies. J Bacteriol 87, 574-580.

Rosan, B. \& Lamont, R. J. (2000). Dental plaque formation. Microbes Infect 2, 1599-1607.

Rozkiewicz, D., Daniluk, T., Zaremba, M. L., Cylwik-Rokicka, D., Luczaj-Cepowicz, E., Milewska, R., Marczuk-Kolada, G. \& Stokowska, W. (2006). Bacterial composition in the supragingival plaques of children with and without dental caries. Adv Med Sci 51 (Suppl. 1), 182-186.

Russell, J. I., MacFarlane, T. W., Aitchison, T. C., Stephen, K. W. \& Burchell, C. K. (1990). Salivary levels of mutans streptococci, Lactobacillus, Candida, and Veillonella species in a group of Scottish adolescents. Community Dent Oral Epidemiol 18, 17-21.

Salema, M., Lolkema, J. S., San Romão, M. V. \& Lourero Dias, M. C. (1996). The proton motive force generated in Leuconostoc oenos by Lmalate fermentation. J Bacteriol 178, 3127-3132.

Schneider, K., Dimroth, P. \& Bott, M. (2000). Biosynthesis of the prosthetic group of citrate lyase. Biochemistry 39, 9438-9450.

Sheng, J. \& Marquis, R. E. (2007). Malolactic fermentation by Streptococcus mutans. FEMS Microbiol Lett 272, 196-201.

Sibley, C. D., Rabin, H. \& Surette, M. G. (2006). Cystic fibrosis: a polymicrobial infectious disease. Future Microbiol 1, 53-61.

Sperandio, B., Gautier, C., Pons, N., Ehrlich, D. S., Renault, P. \& Guédon, E. (2010). Three paralogous LysR-type transcriptional regulators control sulfur amino acid supply in Streptococcus mutans. J Bacteriol 192, 3464-3473.

Terleckyj, B. \& Shockman, G. D. (1975). Amino acid requirements of Streptococcus mutans and other oral streptococci. Infect Immun 11, 656-664.

Thomas, E. L., Pera, K. A., Smith, K. W. \& Chwang, A. K. (1983). Inhibition of Streptococcus mutans by the lactoperoxidase antimicrobial system. Infect Immun 39, 767-778.

Tong, H., Chen, W., Merritt, J., Qi, F., Shi, W. \& Dong, X. (2007). Streptococcus oligofermentans inhibits Streptococcus mutans through conversion of lactic acid into inhibitory $\mathrm{H}_{2} \mathrm{O}_{2}$ : a possible counteroffensive strategy for interspecies competition. Mol Microbiol 63, 872880.

van der Ploeg, J. R. (2005). Regulation of bacteriocin production in Streptococcus mutans by the quorum-sensing system required for development of genetic competence. J Bacteriol 187, 3980-3989.

Wang, B. Y. \& Kuramitsu, H. K. (2005). Interactions between oral bacteria: inhibition of Streptococcus mutans bacteriocin production by Streptococcus gordonii. Appl Environ Microbiol 71, 354-362.

Wu, C., Ayala, E. A., Downey, J. S., Merritt, J., Goodman, S. D. \& Qi, F. (2010a). Regulation of ciaXRH operon expression and identification of the CiaR regulon in Streptococcus mutans. J Bacteriol 192, 46694679 .

Wu, C., Cichewicz, R., Li, Y., Liu, J., Roe, B., Ferretti, J., Merritt, J. \& Qi, F. (2010b). Genomic island TnSmu2 of Streptococcus mutans harbors a nonribosomal peptide synthetase-polyketide synthase gene cluster responsible for the biosynthesis of pigments involved in oxygen and $\mathrm{H}_{2} \mathrm{O}_{2}$ tolerance. Appl Environ Microbiol 76, 5815-5826.

Xie, Z., Okinaga, T., Niu, G., Qi, F. \& Merritt, J. (2010). Identification of a novel bacteriocin regulatory system in Streptococcus mutans. Mol Microbiol 78, 1431-1447.

Zaura, E., Keijser, B. J., Huse, S. M. \& Crielaard, W. (2009). Defining the healthy "core microbiome" of oral microbial communities. BMC Microbiol 9, 259.

Edited by: M. Whiteley 